\title{
Efectividad del cipionato de estradiol inyectado al final de un tratamiento con progesterona sobre la eficiencia reproductiva*
}

\author{
Uslenghi, G.; Chayer, R. y Callejas, S. \\ Área de Reproducción, Facultad de Ciencias Veterinarias, Universidad Nacional del Centro de la Provincia de \\ Buenos Aires. Paraje Arroyo Seco s/n. Tandil (7000), Argentina. Tel.: 02293-439850. \\ E-mail: callejas@vet.unicen.edu.ar
}

\begin{abstract}
Resumen
Uslenghi, G.; Chayer, R. y Callejas, S.: Efectividad del cipionato de estradiol inyectado al final de un tratamiento con progesterona sobre la eficiencia reproductiva. Rev. vet. 21: 1, 55-58, 2010. El cipionato de estradiol (CPE) ha sido utilizado para reemplazar al benzoato de estradiol (BE) administrado vía IM $24 \mathrm{~h}$ después de retirado el dispositivo intravaginal con progesterona (DISP), sin afectar los porcentajes de preñez. El objetivo del trabajo fue evaluar el efecto de dos dosis de CPE inyectado al retirar un DISP, sobre el porcentaje de preñez post inseminación artificial a tiempo fijo (IATF). Como objetivo secundario, se planteó comparar la eficiencia reproductiva de los tratamientos utilizados. Se emplearon 79 vaquillonas de 15 meses de edad. El día 0, se les colocó un DISP más BE. El día 7 se retiró el DISP, se administró $\mathrm{D}(+)$ cloprostenol y en forma aleatoria se inyectó $0,5 \mathrm{mg}$ de CPE, $1 \mathrm{mg}$ de CPE o BE 24 h después. Para la IATF (52-56 h) se utilizó semen proveniente de un toro de probada fertilidad. El día 22 se colocó a todas las vaquillonas un DISP de segundo uso, retirándolo 7 días después. Se continuó con detección de celo e IA. El diagnóstico de gestación se realizó por ultrasonografía en dos oportunidades para evaluar preñez de IATF y retorno, así como para verificar mortalidad embrionaria. Se evaluó el efecto de los tratamientos sobre la preñez a la IATF, del retorno y final. No se observaron efectos del tratamiento $(\mathrm{p}>0,05)$ sobre los porcentajes de preñez a la IATF $(51,9 \%)$, del retorno $(61,1 \%)$ y final $(65,8 \%)$. Se observó un $7,3 \%$ de pérdidas embrionarias ( $p>0,05$ ). Se concluye que la administración de 0,5 o $1 \mathrm{mg}$ de $\mathrm{CPE}$ al retirar un DISP con progesterona en lugar de BE a las $24 \mathrm{~h}$ posteriores es igualmente efectiva para implementar una IATF. Además, no resulta afectada la eficiencia reproductiva del rodeo.
\end{abstract}

Palabras clave: vaquillona, cipionato de estradiol, inseminación artificial a tiempo fijo, tasa de preñez.

\begin{abstract}
Uslenghi, G.; Chayer, R. y Callejas, S.: Effectiveness of estradiol cypionate administered at the end of progesterone treatment upon reproductive efficiency. Rev. vet. 21: 1, 55-58, 2010. Estradiol cypionate (CPE) has been used at the time of progesterone intravaginal device removal to replace the administration of estradiol benzoate (BE) $24 \mathrm{~h}$ later, without affecting pregnancy rates. The objective of this work was to evaluate the effect of the administration of two different doses of CPE, at the time of device removal, upon pregnancy rate after fixed-time artificial insemination (FTAI). Reproductive efficiency of both treatments was investigated as well. Seventy-nine Aberdeen Angus heifers, 15 month-old, were used. On day 0 , intravaginal device was fitted and estradiol benzoate injected; on day 7 , device was removed, $\mathrm{D}(+)$ cloprostenol was injected and heifers were randomly assigned to receive 0.5 $\mathrm{mg}$ of CPE, $1 \mathrm{mg}$ of CPE or EB $24 \mathrm{~h}$ later. FTAI was performed 52-56 h after device removal, using semen from a bull of proven fertility. On day 22, a second-hand intravaginal device was fitted for 7 days. Treatment continued with estrus detection and AI. Pregnancy diagnosis was performed twice to evaluate the pregnancy rate of FTAI, estrus return and embryo death rate. The effect of different treatments upon pregnancy rates of FTAI, estrus return and final pregnancy rate were evaluated. There was no effect of different treatments on pregnancy rates ( $\mathrm{p}>0.05)$ : FTAI (51.9\%), estrus return (61.1\%) and final pregnancy $(65.8 \%)$. A $7.3 \% \mathrm{em}-$ bryo death rate was observed without differences among treatments $(\mathrm{p}>0.05)$. In conclusion,
\end{abstract}

Recibido: 5 abril 2010 / Aceptado: 7 mayo 2010

*Beca de Entrenamiento del primer autor, Comisión de Investigaciones Científicas de la Provincia de Buenos Aires, Argentina. Trabajo presentado en el VIII Simposio Internacional de Reproducción Animal, Córdoba (Argentina), 2009. 
the use of 0.5 or $1 \mathrm{mg}$ of estradiol cypionate at the time of removal of progesterone intravaginal device instead of estradiol benzoate, $24 \mathrm{~h}$ later, can be implemented without affecting the pregnancy rates after FTAI. Furthermore, reproductive efficiency is not affected.

Key words: heifer, estradiol cypionate, fixed-time artificial insemination, pregnancy rate.

\section{INTRODUCCIÓN}

La inseminación artificial es una técnica que permite mejorar genéticamente el rodeo. El control farmacológico del ciclo estral resulta de interés debido a que facilita la implementación de programas de inseminación artificial en los rodeos de cría, pudiendo inseminarse todos los animales en un tiempo prefijado (IATF), con la consiguiente mejora productiva.

Para la realización de la IATF, es fundamental controlar el ciclo estral de los animales a través del uso de diferentes protocolos hormonales, de los cuales uno de los más conocidos es el que utiliza dispositivos intravaginales con progesterona combinado con prostaglandina administrada en el momento de retirarlos y estrógenos inyectados al colocar el dispositivo y $24 \mathrm{~h}$ post retiro de los mismos ${ }^{2}$. Esta técnica está fundada en que el dispositivo intravaginal simula la función de un cuerpo lúteo de corta vida media, de ahí su permanencia durante siete $\mathrm{u}$ ocho días en el tracto vaginal. La inyección de estrógenos al inicio del tratamiento genera una nueva onda de crecimiento folicular, para que, al momento de retirar el dispositivo exista un folículo dominante joven y en crecimiento, con capacidad de ovular, obteniendo un ovocito capaz de ser fertilizado al momento de la inseminación.

La aplicación de prostaglandina o sus análogos sintéticos genera, en el día de extracción del dispositivo, la luteólisis de algún cuerpo lúteo que pudiera existir, con el consiguiente descenso de los niveles de progesterona plasmáticos. Por último, la inyección de estrógenos a las $24 \mathrm{~h}$ post retiro de los dispositivos genera la manifestación de celo por parte de los animales e induce el pico preovulatorio de LH necesario para la ovulación del folículo dominante generado de la onda de crecimiento folicular. El tratamiento descrito permite sincronizar las ovulaciones de los animales cíclicos e inducir actividad sexual en animales en anestro, generando las condiciones para implementar programas de IATF.

La implementación de los protocolos de control del ciclo estral requiere un gran número de encierre de animales, que en algunos casos es causa de estrés severo si no se toman ciertos recaudos, con la consecuente disminución de la fertilidad y fracaso de la técnica. En tal sentido resulta de interés estudiar otros protocolos que disminuyan la cantidad de encierres necesarios, por lo cual se recurrió a la utilización del cipionato de estradiol (CPE) inyectado en el momento de retirar el dispositivo en reemplazo del benzoato de estradiol que se administra $24 \mathrm{~h}$ posteriores ${ }^{3}$.
El objetivo de este trabajo fue evaluar el efecto de dos diferentes dosis de CPE administradas en el momento de retirar un dispositivo intravaginal de progesterona, sobre el porcentaje de preñez que se obtiene luego de realizar una IATF en vaquillonas Aberdeen Angus de 15 meses de edad. Como objetivo secundario, se planteó comparar la eficiencia reproductiva de los diferentes tratamientos utilizados.

\section{MATERIAL Y MÉTODOS}

Lugar, animales. El trabajo se realizó en un establecimiento, ubicado en Oriente, Partido de Coronel Dorrego, Provincia de Buenos Aires, Argentina. Se utilizaron 79 vaquillonas con una edad de 15 meses, Aberdeen Angus y Careta, con una condición corporal promedio de 2,8 $\pm 0,4$ (escala 1 a 5). Todas las vaquillonas fueron seleccionadas de un grupo mayor de animales. En primer lugar se consideró el biotipo y posteriormente se tuvo en cuenta el peso corporal. Para ser seleccionados, los animales debían tener un peso mínimo de $280 \mathrm{~kg}$ (65\% del peso adulto del rodeo).

Alimentación. Los animales se alimentaron sobre pasturas consociadas con base alfalfa en todo momento, tanto antes, como durante y después del tratamiento, recibiendo suplementación con grano de maíz y rollos de avena tres meses previos al inicio del protocolo de sincronización (invierno) con el objetivo de balancear la dieta, ya que en esa estación del año la oferta forrajera de esta pastura disminuye drásticamente.

Tratamientos y servicio. IATF. En el día 0 se colocó a las vaquillonas un dispositivo intravaginal con $1 \mathrm{~g}$ de progesterona (DIB, Syntex), más una inyección intramuscular de $2 \mathrm{mg}$ de benzoato de estradiol (Syntex). El día 7, se retiró el dispositivo y se administró en forma intramuscular $500 \mu \mathrm{g}$ de $\mathrm{D}(+)$ cloprostenol (Ciclase DL, Syntex). En este momento, los animales se dividieron aleatoriamente en tres grupos que recibieron los siguientes tratamientos:

Grupo CPE05 ( $\mathrm{n}=26)$ : recibieron vía intramuscular $0,5 \mathrm{mg}$ de CPE.

Grupo CPE1 $(\mathrm{n}=26)$ : recibieron vía intramuscular 1 $\mathrm{mg}$ de CPE.

Grupo BE24 ( $\mathrm{n}=27)$ : recibieron una inyección intramuscular de $1 \mathrm{mg}$ de BE 24 h después (día 8 del tratamiento).

Todos los animales fueron pintados en la base de la cola para la detección de celos, con el objetivo de realizar la lectura del grado de despintado en el momento 
de la IATF. El día 9, se realizó la IATF (52-56 h post retiro del dispositivo intravaginal), utilizándose semen congelado/descongelado en pajuelas de $0,5 \mathrm{ml}$ provenientes de un toro de probada fertilidad.

Sincronización del retorno. En el día 22 de iniciado el tratamiento (13 días post IATF), se colocó a todas las vaquillonas un dispositivo intravaginal de segundo uso (DIB) con el objetivo de resincronizar los celos, estos dispositivos fueron retirados 7 días después. Durante los días 30 a 33 del tratamiento se realizó detección de celo a campo e inseminación artificial según la regla $\mathrm{am} / \mathrm{pm}$. Se utilizó semen congelado/descongelado en pajuelas de $0,5 \mathrm{ml}$ proveniente de un toro de probada fertilidad.

Diagnóstico de gestación. Se realizó mediante ultrasonografía (transductor sectorial 7,5 MHz; Tringa, Inmed) a los 38 días post IATF para evaluar preñez de la IATF y a los 72 días para evaluar preñez del retorno y si ocurrió mortalidad embrionaria de las preñadas a la IATF.

Análisis estadístico. Se evaluó el efecto de los tratamientos (CPE05, CPE1 o BE24) sobre los porcentajes de preñez a la IATF, del retorno (preñadas en el retorno/ vacías a la IATF) y final (IATF + retorno), utilizando el Proc CATMOD del SAS. Se fijó un nivel de confianza del $95 \%(\alpha=0,05)$.

\section{RESULTADOS}

No se observaron efectos del tratamiento $(p>0,05)$ sobre los porcentajes de preñez en la IATF (Tabla 1), de retorno al celo y de preñez del retorno (Tabla 2) y de preñez final (Tabla 3). Las pérdidas embrionarias encontradas fueron del $7,3 \%$, sin observarse diferencias entre los tratamientos $(\mathrm{p}>0,05)$.

\section{DISCUSIÓN}

El porcentaje de preñez obtenido luego de realizar la IATF es coincidente con lo informado por otros autores ${ }^{11}$; asimismo se encuentra dentro del rango de preñez que cita la bibliografía para la implementación de estos programas de control del ciclo estral, de $33,9 \%$ al $65,4 \%$, con un promedio del $50,0 \%$, 6, 7-10 .

La cantidad de animales que retornaron al celo sobre el total de vaquillonas vacías a la IATF (detectadas como vacías en la ecografía del día 38) no mostró diferencias significativas entre grupos. Si bien este resultado es menor al informado por algunos autores que obtuvieron valores de $52,4 \%$ y $88,9 \%$ respectivamente 9,10 , se encuentra dentro del rango citado por otros que lograron porcentajes de retorno al celo del $40,8 \%$ en vaquillonas de 18 meses y de $68,2 \%$ en vaquillonas de 24 meses $^{1}$.

El porcentaje de preñez del retorno fue superior al obtenido por investigadores que informaron un $36,4 \%$
Tabla 1. Tasas de preñez post IATF según tratamiento.

\begin{tabular}{cc}
\hline tratamiento & preñez a la IATF $(\%)$ \\
\hline CPE05 & $50,0(13 / 26)$ \\
CPE1 & $46,2(12 / 26)$ \\
BE24 & $59,3(16 / 27)$ \\
\hline
\end{tabular}

CPE05 y CPE1: 0,5 y $1 \mathrm{mg}$ de cipionato de estradiol respectivamente, BE24: benzoato de estradiol.

Tabla 2. Tasas de retorno al celo y preñez al retorno, según tratamiento.

\begin{tabular}{ccc}
\hline tratamiento & retorno al celo (\%) & preñez del retorno (\%) \\
\hline CPE05 & $61,5(8 / 13)$ & $62,5(5 / 8)$ \\
CPE1 & $50,0(7 / 14)$ & $57,1(4 / 7)$ \\
BE24 & $27,3(3 / 11)$ & $66,7(2 / 3)$ \\
\hline
\end{tabular}

CPE05 y CPE1: 0,5 y $1 \mathrm{mg}$ de cipionato de estradiol respectivamente, BE24: benzoato de estradiol.

Tabla 3. Porcentajes de preñez final (IATF + retorno), según tratamiento.

\begin{tabular}{cc}
\hline tratamiento & preñez final (\%) \\
\hline CPE05 & $69,2(18 / 26)$ \\
CPE1 & $61,5(16 / 26)$ \\
BE24 & $66,7(18 / 27)$ \\
\hline
\end{tabular}

CPE05 y CPE1: 0,5 y $1 \mathrm{mg}$ de cipionato de estradiol respectivamente, BE24: benzoato de estradiol.

de preñez de las vaquillonas que retornaron al celo luego de realizada una IATF ${ }^{9}$. No obstante, en otros trabajos se ha informado un rango de 50 a $75 \%$ en vaquillonas de 15 meses ${ }^{10} \mathrm{y}$ de 35 a $66,7 \%$ en vaquillonas de 18 a 24 meses ${ }^{1}$.

El porcentaje de preñez final $(65,8 \%)$ coincide con la tasa obtenida en otro trabajo, de $67,9 \%{ }^{9}$. Por otra parte, supera al valor obtenido en vaquillonas de 18 meses, donde se obtuvo $56,4 \%$ de preñez final, pero es menor cuando se compara al obtenido en vaquillonas de 24 meses donde dicho porcentaje fue de $80,3 \%{ }^{1}$. Además, se encuentra dentro del rango citado por investigadores que trabajando con semen de dos toros, obtuvieron porcentajes que variaron de 64 a $88,5 \%$ de preñez final en vaquillonas de 15 meses ${ }^{10}$. Diferencias en las condiciones ambientales, inseminadores, semen utilizado y otras variables no medidas, podrían explicar las diferencias observadas entre autores. El porcentaje de pérdidas embrionarias (7,3\%) fue superior al hallado en otros trabajos, de 2,9 a $4,8 \%{ }^{5,12}$, no encontrándose una explicación a dichas divergencias. Un análisis sobre mayor número de animales debería clarificar este aspecto.

Se concluye que la administración de $0,5 \mathrm{mg}$ o $1 \mathrm{mg}$ de CPE en el momento de retirar un dispositivo intravaginal con $1 \mathrm{~g}$ de progesterona en lugar de benzoato de estradiol a las $24 \mathrm{hs}$ posteriores, es igualmente efectiva para implementar un protocolo de IATF en vaquillonas 
de 15 meses de edad. Además, no se afecta la eficiencia reproductiva del rodeo.

\section{REFERENCIAS}

1. Balla E, Maraña Peña $P$, Chesta $P$, Pincinato $D$, Tríbulo R, Bó GA. 2005. Efecto de dos dosis de BE en el momento de la reinserción del CIDr-B en un programa de resincronización de celos en vaquillonas. Anales VI Simposio Internacional de Reproducción Animal, Córdoba (Argentina), p. 388.

2. Callejas S. 2005. Control farmacológico del ciclo estral bovino: bases fisiológicas, protocolos y resultados. Parte II. Rev Taurus 25: 16-35.

3. Callejas S, De Dominici O, Madero S, Cantallops F, Cabodevila J. 2005. Efecto del CPE administrado al momento de retirar un dispositivo intravaginal con progesterona o 24 hs después sobre el porcentaje de preñez a la IATF. Anales VI Simposio Internacional de Reproducción Animal, Córdoba (Argentina), p. 391.

4. Cesaroni G, Butler HM, Durand MJ. 2007. Evaluación del uso de dos ésteres de estradiol sobre la tasa de fertilidad a la IATF en vacas secas, tratadas con un dispositivo intravaginal con progesterona. Anales VII Simposio Internacional de Reproducción Animal, Córdoba (Argentina), p. 242.

5. De Dominici O, Callejas S. 2004. Fertilidad de la IATF realizada después de la administración de benzoato de estradiol o de GnRH al final de un tratamiento con progesterona/progestágenos en vacas para carne. Rev Arg Prod Anim 24 (Supl. 1): 280-281.

6. De Dominici O, Madero S, Cantallops F, Callejas S. 2007. Efecto de la eCG administrada al final de un tratamiento de sincronización de celos con progesterona sobre el porcentaje de preñez a la IATF en vaquillonas para carne. Anales VII Simposio Internacional de Reproducción Animal, Córdoba (Argentina), p. 227.
7. González Chaves S, Chayer R, Callejas S. 2007. Efecto de administrar media dosis de un agente luteolítico al momento de colocar y retirar un dispositivo intravaginal con 0,558 g de progesterona sobre el porcentaje de preñez a la IATF en vaquillonas para cría. Anales VII Simposio Internacional de Reproducción Animal, Córdoba (Argentina), p. 230 .

8. González Chaves S, Chayer R, Catalano R, Cabodevila J, Callejas S. 2007. Efecto de administrar media dosis de un agente luteolítico al momento de colocar y retirar un dispositivo intravaginal con $1 \mathrm{~g}$ de progesterona y de la duración del tratamiento sobre el porcentaje de preñez a la IATF en vaquillonas angus. Anales VII Simposio Internacional de Reproducción Animal, Córdoba (Argentina), p. 232.

9. Madero S, De Dominici O, Cantallops F, Catalano R, Callejas S. 2007. Resincronización de celos en vaquillonas Angus: uso de 0,5 mg de benzoato de estradiol en el momento de colocar un dispositivo intravaginal con progesterona. Anales VII Simposio Internacional de Reproducción Animal, Córdoba (Argentina), p. 223.

10. Madero S, De Dominici O, Cantallops F, Uslenghi G, Callejas S. 2007. Resincronización de celos en vaquillonas Angus: uso de 0,7 mg de benzoato de estradiol en el momento de colocar un dispositivo intravaginal con progesterona. Anales VII Simposio Internacional de Reproducción Animal, Córdoba (Argentina), p. 225.

11. Madero S, De Dominici O, Cantallops F, Uslenghi G, Callejas S. 2007. Efecto de dos dosis de CPE administradas al finalizar un tratamiento con dispositivos intravaginales con progesterona sobre el porcentaje de preñez a la IATF. Anales VII Simposio Internacional de Reproducción Animal, Córdoba (Argentina), p. 226.

12. Santos JE, Thatcher WW, Chebel RC, Cerri RL, Galvao RN. 2004. The effect of embryonic death rate in cattle on the efficacy of estrus synchronization programs. Anim Reprod Sci 82/83: 513-535. 